\title{
Conditional symmetry and spectrum of the one-dimensional Schrödinger equation
}

\author{
R.Z. Zhdanov \\ Arnold-Sommerfeld Institute for Mathematical Physics, \\ Leibnitzstraße 10, 38678 Clausthal-Zellerfeld, Germany \\ Electronic mail asrz@pt.tu-clausthal.de *
}

\begin{abstract}
We develop an algebraic approach to studying the spectral properties of the stationary Schrödinger equation in one dimension based on its high order conditional symmetries. This approach makes it possible to obtain in explicit form representations of the Schrödinger operator by $n \times n$ matrices for any $n \in \mathbf{N}$ and, thus, to reduce a spectral problem to a purely algebraic one of finding eigenvalues of constant $n \times n$ matrices. The connection to so called quasi exactly solvable models is discussed. It is established, in particular, that the case, when conditional symmetries reduce to high order Lie symmetries, corresponds to exactly solvable Schrödinger equations. A symmetry classification of Schödinger equation admitting non-trivial high order Lie symmetries is carried out, which yields a hierarchy of exactly solvable Schrödinger equations. Exact solutions of these are constructed in explicit form. Possible applications of the technique developed to multi-dimensional linear and one-dimensional nonlinear Schrödinger equations is briefly discussed.
\end{abstract}

${ }^{*}$ On leave from the Institute of Mathematics of the Academy of Sciences of Ukraine, Tereshchenkivska Str.3, 252004 Kiev, Ukraine 


\section{Introduction}

Basic motivation for introducing conditional symmetries (the term 'conditional symmetry' was suggested for the first time by FusHCHYCH [1]-[3]) was a necessity to find a symmetry background of a quickly growing variety of exact solutions of nonlinear partial differential equations that could not be obtained within the framework of the classical Lie approach. An intensive search of such solutions was begun independently and almost simultaneously by FusHCHYCH with collaborators (see, [4, 5] and references therein), Clarkson \& Kruskal ('the direct reduction method' [6]), Olver \& Rosenau ('non-classical reduction' [7]) and Winternitz \& LEVi [8]. A number of examples of nonlinear partial differential equations in two, three and even four dimensions having non-trivial conditional symmetries is growing rapidly. In particular, it has been established by FusHCHYCH, ZHDANOV AND REVENKO [9]-13 that such fundamental equations of the modern quantum field theory as the four-dimensional nonlinear d'Alembert, Dirac, LeviLeblond, Maxwell and Yang-Mills equations possess infinite conditional symmetries, while their Lie symmetries are finite only.

On the other hand, much less attention is devoted to the study of conditional symmetries of linear differential equations (though the first example of conditional symmetry has been obtained by BLUMAN and COLE for the one-dimensional linear heat equation [14]). In view of the role played by conditional symmetries in the theory of nonlinear differential equations one can expect that application of these to linear equations will also be rich in results. In the present paper we establish the rather unexpected (at least for the author) fact that conditional symmetries can be effectively applied to study spectral properties of the stationary Schrödinger equation

$$
\psi_{x x}=(\varepsilon+V(x)) \psi .
$$

In particular, we will prove that it is conditional symmetry that is responsible for a phenomenon of so called 'quasi exact solvability' of some specific

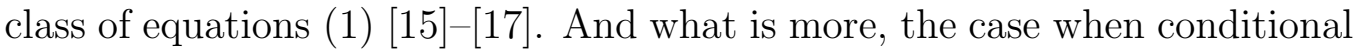
symmetries are equivalent to Lie symmetries will be shown to yield exactly solvable Schrödinger equations.

All principal approaches to a construction of (quasi) exactly solvable models (apart from the specific ways of an implementation of these) are based on a possibility to construct a basis $u_{1}(x), u_{2}(x), \ldots, u_{n}(x)$ of some invariant 
space $\mathcal{V}_{n}$ of the Schrödinger operator $S=\partial_{x}^{2}-V(x)$. This means that there should exist constant $n \times n$ matrix $\left\|\Lambda_{j k}\right\|$ such that the following conditions are fulfilled:

$$
S u_{j}(x) \equiv\left(\partial_{x}^{2}-V(x)\right) u_{j}(x)=\sum_{k=1}^{n} \Lambda_{j k} u_{k}(x), \quad j=1,2, \ldots, n
$$

Given such functions $u_{j}(x)$, a procedure for calculating the spectrum of the Schrödinger operators (or, more precisely, a part of the spectrum) is completely algebraic. Let $\vec{a}^{j}=\left(a_{1}^{j}, a_{2}^{j}, \ldots, a_{n}^{j}\right), j=1, \ldots, m, m \leq n$ be a complete system of eigenvectors of the $(n \times n)$ matrix $\Lambda=\left\|\Lambda_{j k}\right\|_{j, k=1}^{n}$ and $\lambda_{1}, \ldots, \lambda_{m}$ be their eigenvalues, namely

$$
\sum_{j=1}^{n} \Lambda_{j k} a_{j}^{l}=\lambda_{l} a_{k}^{l}, \quad j=1,2, \ldots, m .
$$

Then, the function

$$
\psi_{k}(x)=\sum_{j=1}^{n} a_{j}^{k} u_{j}(x)
$$

is easily seen to satisfy the equation (11) with $\varepsilon=\lambda_{k}$ under arbitrary $k=$ $1,2, \ldots, m$.

Saying it another way, after being restricted to a linear space $\mathcal{V}_{n}$ with basis functions $u_{1}(x), u_{2}(x), \ldots, u_{n}(x)$ the Schrödinger operator becomes a matrix operator. Thus, a reduction of a differential operator to a matrix operator takes place. But such a procedure is quite a common routine in the theory of Lie symmetries of differential equations. Indeed, if we restrict a partial differential equation having $N$ independent variables to a subset of its solutions invariant under a one-parameter subgroup of the Lie group admitted by the equation in question, then it is reduced to a partial differential equation with $N-1$ independent variables. Such a procedure is called symmetry reduction of differential equations (for more detail, see e.g. [4, 18, 19]). Taking $N=1$ (the case of ordinary differential equation) we obtain as a reduced equation a differential equation with $N=0$, i.e. an algebraic equation!

One of the main aims of the present paper is to show that the idea of symmetry reduction, when formulated in an appropriate way, can be applied effectively to an algebraization of the problem of describing spectrum of the Schrödinger operator. 
As mentioned above classical Lie symmetries of partial differential equation do not give all possible reductions. More general symmetries responsible for a possibility of reducing the order of differential equations are conditional symmetries. Roughly speaking, the necessary and sufficient condition providing a possibility to reduce a number of variables in a given partial differential equation is a requirement of conditional invariance ([20]). It will be established that a similar situation takes place for the Schrödinger equation (1). Symmetries providing reducibility of differential equation (1) to a system of algebraic equations of the form (2) are exactly the high order conditional symmetries introduced independently by ZHDANOV \& FUSHCHYCH 21, 22] and FoKAs \& LIU 23] (see also [24]-26]).

It should be emphasized that considerations of the present paper are purely algebraic. The method of conditional symmetries making it possible to study spectral properties of the Schrödinger operator $S$ gives no information about analytical properties of the corresponding eigenfunctions. In each specific case such properties of the eigenfunctions obtained as square integrability, asymptotic behavior, singularities etc. should be studied independently (see, e.g. [27]). The reason is that this method (and group-theoretical, symmetry methods in general) exploits algebraic properties of the solution set of equation (1) (or its part) as a whole and, roughly speaking, is independent of analytical properties of specific solutions.

\section{Conditional symmetry of the Schrödinger equation}

Consider the $n$-th order differential operator

$$
Q=\sum_{j=0}^{n} q_{j}(x) \partial_{x}^{j}
$$

where $\partial_{x}^{0}=1, \partial_{x}^{j+1}=\frac{d}{d x} \partial_{x}^{j}$ and functions $q_{j}(x)$ are supposed to be independent of $\varepsilon$.

Following [28, 29] we say that equation (11) is conditionally invariant with respect to the operator $Q$ if the following operator identity holds:

$$
\left[Q, \partial_{x}^{2}-(\varepsilon+V(x))\right]=R Q+P\left(\partial_{x}^{2}-(\varepsilon+V(x)) .\right.
$$


Here $\left[Q_{1}, Q_{2}\right] \equiv Q_{1} Q_{2}-Q_{2} Q_{1}, R, P$ are some first and $n$-th order differential operators, correspondingly. The above operator equality should be understood in the following way: the differential operators in the left- and right-hand sides give the same result when acting on arbitrary $(n+2)$-times continuously differentiable function $f(x)$.

Provided $R$ vanishes, condition (6) is nothing else but a criterion for equation (1) to be invariant with respect to the operator $Q$. In such a case, the operator $Q$ is a generalized (high order) Lie symmetry operator. But given a condition $R \neq 0$, the operator $Q$ corresponds to high order conditional symmetry of the Schrödinger equation (11).

It is easy to see that if an equation is conditionally invariant with respect to the operator $Q$, then it is conditionally invariant with respect to the operator $q(x) Q$ with an arbitrary sufficiently smooth function $q(x)$. Consequently, without loss of generality we can suppose that in (5) $q_{n}(x)=1$ and consider differential operators of the form

$$
Q=\partial_{x}^{n}+\sum_{j=0}^{n-1} q_{j}(x) \partial_{x}^{j} .
$$

As the coefficients of the operator $Q$ do not depend on $\varepsilon$, equality (6) is only possible if $R=r(x)$ and $P=0$ with some sufficiently smooth function $r(x)$. Consequently, the condition (6) is rewritten to become

$$
\left[Q, \partial_{x}^{2}-V(x)\right]=r(x) Q .
$$

We call the Schrödinger operator reducible if there exist linearly-independent functions $u_{1}(x), \ldots, u_{n}(x)$ and constants $\Lambda_{j k}$ such that the conditions (2) are fulfilled. Let us note that this terminology is justified both from the point of view of the classical representation theory and of the symmetry analysis of differential equations. Indeed, conditions (2) mean that the representation space of the operator $S$ contains an invariant subspace and, consequently the representation is reducible. On the other hand, conditions (2) ensure the reduction of the differential equation (1) to a system of algebraic equations. We will prove an assertion which shows that this is not a simple coincidence but a fundamental fact having a natural symmetry interpretation.

Theorem 1 The Schrödinger operator $S=\partial_{x}^{2}-V(x)$ is reducible if and only if there exists an $n$-th order differential operator $Q$ of the form (5) such that equation (1) is conditionally invariant with respect to $Q$. 
Proof. The necessity. Let the operator $S=\partial_{x}^{2}-V(x)$ be reducible. Then, the conditions (21) hold. As functions $u_{j}(x)$ are linearly independent, they form a fundamental system of solutions of some $n$-th order linear ordinary differential equation [30].

We recall that fundamental system of solutions of an ordinary differential equation is a maximal set of its particular solutions such that any smooth solution can be represented as a linear combination of these. Provided the order of the ordinary differential equation in question is equal to $n$, any $n$ linearly independent solutions of it form a fundamental system. Furthermore, having a fundamental system of solutions we can reconstruct the corresponding ordinary differential equation within a multiplication by a function $r(x)$. Consequently, if we fix the coefficient of the $n$-th order derivative to be equal to 1 , then this equation is unique.

Thus, there exists the $n$-th order differential equation

$$
u^{(n)}(x)+\sum_{j=0}^{n-1} \tilde{q}_{j}(x) u^{(j)}(x)=0,
$$

such that the functions $u_{j}(x)$ form a fundamental system of its solutions.

We will prove that equation (1) is conditionally invariant with respect to the operator

$$
\tilde{Q}=\partial_{x}^{n}+\sum_{j=0}^{n-1} \tilde{q}_{j}(x) \partial_{x}^{j} .
$$

By force of relations (22) the following equalities hold:

$$
\begin{aligned}
& {\left[\tilde{Q}, \partial_{x}^{2}-V(x)\right] u_{j}(x)=\tilde{Q}\left\{\left(\partial_{x}^{2}-V(x)\right) u_{j}(x)\right\}} \\
& \quad-\left(\partial_{x}^{2}-V(x)\right)\left\{\tilde{Q} u_{j}(x)\right\}=\tilde{Q}\left\{\sum_{k=1}^{n} \Lambda_{j k} u_{k}(x)\right\}=0
\end{aligned}
$$

for any $j=1,2, \ldots, n$.

Thus, the functions $u_{j}(x)$ satisfy an ordinary differential equation

$$
\left[\tilde{Q}, \partial_{x}^{2}-(\varepsilon+V(x))\right] u(x)=0,
$$

whose order is easily established to be equal to $n$. Consequently, its fundamental system of solutions consists of $n$ functions. Hence, we conclude that the functions $u_{j}(x)$ form a fundamental system of solutions of (10). As 
an ordinary differential equation is determined by its fundamental system uniquely within a multiplication by a function $r(x)$, the relation hold

$$
\left[\tilde{Q}, \partial_{x}^{2}-(\varepsilon+V(x))\right]=r(x) \tilde{Q},
$$

which is the same as what was to be proved.

The sufficiency. Let the Schrödinger equation (1) be conditionally invariant with respect to the operator (7), which means that the condition (8) is fulfilled. Consider an equation

$$
Q u(x) \equiv\left(\partial_{x}^{n}+\sum_{j=0}^{n-1} q_{j}(x) \partial_{x}^{j}\right) u(x)=0
$$

as an ordinary differential equation for a function $u(x)$. Clearly, its general solution is represented in the form

$$
u(x)=\sum_{j=1}^{n} C_{j} u_{j}(x),
$$

where $C_{j}$ are arbitrary constants and $u_{1}(x), \ldots, u_{n}(x)$ is a fundamental system of solutions of $n$-th order ordinary differential equation (11).

From the condition (8) it follows that the Schrödinger operator $S=$ $\partial_{x}^{2}-V(x)$ is a symmetry operator for the equation (11), i.e. it transforms each solution of equation (11) into another solution of the same equation. Consequently, for any $j=1,2, \ldots, n$ the function $\tilde{u}_{j}(x)=\left(\partial_{x}^{2}-V(x)\right) u_{j}(x)$ satisfy (11). But by definition the fundamental system of solutions of $n$-th order ordinary differential equation forms a maximal set of its linearly independent solutions, which means that any solution can be represented as a linear combination of functions $u_{j}(x)$. Thus, there exist such constants $\Lambda_{j k}$ that functions $u_{j}(x)$ satisfy relations (2), whence it follows that the corresponding Schrödinger equation is reducible. The theorem is proved.

Note that the proof of theorem is, in fact, independent of the specific form of the Schrödinger operator $S=\partial_{x}^{2}-V(x)$. It is straightforward to generalize Theorem 1 to the case of an arbitrary $N$-th order differential operator

$$
\tilde{S}=\sum_{j=0}^{N} f_{j}(x) \partial_{x}^{j}
$$

We give the corresponding assertion without proof. 
Theorem 2 The operator $\tilde{S}$ in (19) is reducible if and only if there exists $n$ th order differential operator $Q$ of the form (5) such that equation $\tilde{S} \psi(x)=0$ is conditionally invariant with respect to $Q$.

To illustrate the above statement we consider two examples.

Example 1. Consider the harmonic oscillator Schrödinger equation

$$
\psi_{x x}=\left(\varepsilon+x^{2}\right) \psi .
$$

As a direct check shows the $n$-th order differential operator

$$
Q=\left(\partial_{x}-x\right)^{n}
$$

satisfies the following commutation relation:

$$
\left[Q, \partial_{x}^{2}-\left(\varepsilon+x^{2}\right)\right]=2 n Q
$$

(the easiest way to prove the above formula is to use the mathematical induction method).

Consequently, equation (14) is conditionally invariant with respect to the operator $Q$ and we can apply Theorem 1. Integrating equation $Q \psi(x)=0$ yields a basis of the invariant space $\mathcal{V}_{n}$ of the Schrödinger operator $\partial_{x}^{2}-x^{2}$

$$
\mathrm{e}^{-\frac{x^{2}}{2}}, \quad x \mathrm{e}^{-\frac{x^{2}}{2}}, \quad x^{2} \mathrm{e}^{-\frac{x^{2}}{2}}, \ldots, \quad x^{n-1} \mathrm{e}^{-\frac{x^{2}}{2}} .
$$

It is readily seen that the above functions satisfy relations (2) with $V(x)=$ $x^{2}$. Calculating eigenvalues $\left(\lambda_{j}\right)$ and eigenvectors $\left(\vec{a}^{j}\right)$ of the corresponding matrix $\left\|\Lambda_{j k}\right\|$ we obtain exact solutions of the Schrödinger equation (14) with $\varepsilon=\lambda_{1}, \ldots, \lambda_{m}$ in the form (四).

Example 2. Let us generalize the previous example as follows. We are looking for the Schrödinger equations (11) conditionally invariant with respect to the $n$-th order operator which can be represented as a power of the first order differential operator, i.e.

$$
Q=\left(a(x) \partial_{x}+b(x)\right)^{n+1}
$$

By an appropriate transformation of the dependent and independent variables

$$
z=F(x), \quad \varphi(z)=\mathrm{e}^{-\int G(x) d x} \psi(x)
$$


we can transform the operator $Q$ as follows:

$$
\tilde{Q}=\partial_{z}^{n+1}
$$

After rewriting the initial Schrödinger equation in the new variables $z, \varphi(z)$ we get

$$
f(z) \varphi_{z z}+g(z) \varphi_{z}+(h(z)-\varepsilon) \varphi=0
$$

where

$$
\begin{aligned}
& f(z)=\left(F^{\prime}(x)\right)^{2}, \quad g(z)=F^{\prime \prime}(x)+2 F^{\prime}(x) G(x), \\
& h(z)=-V(x)+G^{\prime}(x)+G^{2}(x) .
\end{aligned}
$$

Commutation relations (8) now read as

$$
\left[\partial_{z}^{n+1}, f(z) \partial_{z}^{2}+g(z) \partial_{z}+h(z)-\varepsilon\right]=r(z) \partial_{z}^{n+1} .
$$

Computing the commutator in the left-hand side (which is a simple exercise in differential calculus) and equating coefficients of the linearly independent operators $\partial_{z}^{j}$ we conclude that the equation (17) is consistent if and only if the functions $f, g, h$ are polynomials in $z$ of the following form:

$$
\begin{aligned}
& h(z)=A_{0}-n\left(B_{2}+(n-1) C_{3}\right) z+C_{4}(n-1) n z^{2} \\
& g(z)=B_{0}+B_{1} z+B_{2} z^{2}+2 C_{4}(1-n) z^{3} \\
& f(z)=C_{0}+C_{1} z+C_{2} z^{2}+C_{3} z^{3}+C_{4} z^{4}
\end{aligned}
$$

where $A_{0}, B_{0}, B_{1}, \ldots, C_{4}$ are arbitrary constants.

Returning back to the initial variables $x, u(x)$ we get the necessary and sufficient conditions for the Schrödinger equation (1) to be conditionally invariant with respect to an operator belonging to the class (16)

$$
\begin{aligned}
& -V+G^{\prime}+G^{2}=A_{0}-n\left(B_{2}+(n-1) C_{3}\right) F+C_{4}(n-1) n F^{2}, \\
& F^{\prime \prime}+2 F^{\prime} G=B_{0}+B_{1} F+B_{2} F^{2}+2 C_{4}(1-n) F^{3}, \\
& \left(F^{\prime}\right)^{2}=C_{0}+C_{1} F+C_{2} F^{2}+C_{3} F^{3}+C_{4} F^{4},
\end{aligned}
$$

whence we derive the form of the potential $V(x)$

$$
V(x)=\frac{v_{0}+v_{1} \omega+v_{2} \omega^{2}+v_{3} \omega^{3}+v_{4} \omega^{4}}{16\left(C_{0}+C_{1} \omega+C_{2} \omega^{2}+C_{3} \omega^{3}+C_{4} \omega^{4}\right)},
$$




$$
\begin{aligned}
v_{0}= & 4 B_{0}^{2}-16 A_{0} C_{0}+8 B_{1} C_{0}-8 B_{0} C_{1}+3 C_{1}^{2}-8 C_{0} C_{2}, \\
v_{1}= & 8 B_{0} B_{1}+16 B_{2} C_{0}(n+1)-16 A_{0} C_{1}-16 B_{0} C_{2}+4 C_{1} C_{2} \\
& +16 B_{2} C_{0} n+8 C_{0} C_{3}\left(2 n^{2}-2 n-3\right), \\
v_{2}= & 4 B_{1}^{2}+8 B_{0} B_{2}+8 B_{2} C_{1}(2 n+1)-16 A_{0} C_{2}-8 B_{1} C_{2}+4 C_{2}^{2} \\
& -24 B_{0} C_{3}+2 C_{1} C_{3}\left(8 n^{2}-8 n-3\right)-16 C_{0} C_{4} n(n+2), \\
v_{3}= & 8 B_{1} B_{2}-16 A_{0} C_{3}-16 B_{1} C_{3}-16 B_{0} C_{4}(n+1)+16 B_{2} C_{2} n \\
& -16 B_{0} C_{4} n-8 C_{1} C_{4}\left(2 n^{2}+2 n-1\right)+4 C_{2} C_{3}\left(4 n^{2}-4 n+1\right), \\
v_{4}= & 4 B_{2}{ }^{2}+8 B_{2} C_{3}(2 n-1)-16 A_{0} C_{4}-8 B_{1} C_{4}(2 n+1)+ \\
& +C_{3}{ }^{2}\left(16 n^{2}-16 n+3\right)+8 C_{2} C_{4}\left(1-2 n^{2}\right)
\end{aligned}
$$

and of the function $G(x)$

$$
G(x)=\frac{2 B_{0}-C_{1}+2\left(B_{1}-C_{2}\right) \omega+\left(2 B_{2}-3 C_{3}\right) \omega^{2}-4 C_{4} n \omega^{3}}{4 \sqrt{C_{0}+C_{1} \omega+C_{2} \omega^{2}+C_{3} \omega^{3}+C_{4} \omega^{4}}} .
$$

In the above formulae $\omega(x)$ is an elliptic function determined by the quadrature

$$
\int^{\omega(x)} \frac{d \tau}{\sqrt{C_{0}+C_{1} \tau+C_{2} \tau^{2}+C_{3} \tau^{3}+C_{4} \tau^{4}}}=x .
$$

Furthermore, exact solutions of the Schrödinger equation with the potential (18) read as

$$
\psi(x)=\mathrm{e}^{\int G(x) d x} \sum_{j=0}^{n} a_{j} \omega(x)^{j},
$$

where $\vec{a}=\left(a_{0}, a_{1}, \ldots, a_{n}\right)$ is an eigenvector of some $(n+1 \times n+1)$ constant matrix whose entries are linear combinations of the parameters $A_{0}, B_{0}, B_{1}, \ldots$, $C_{4}$ (we omit the corresponding formulae).

Thus, we arrived at the nine-parameter family of quasi exactly solvable Schrödinger equations obtained by TURBINER and SHIFMAN within the framework of their Lie algebraic approach [15, 16] and by UsHVERIDZE by means of a more general analytic approach. A detailed account of properties of the Schrödinger equation with potentials (18) can be found in the monograph [17. We restrict ourselves to noting that if we choose in the above formulae $B_{2}=C_{3}=C_{4}=0$, then the potential $V(x)$ does not depend on $n$ (the order of the operator $Q$ ) and, consequently, the corresponding 
Schrödinger equation is exactly solvable. Thus, the well-known six-parameter family of exactly solvable Schrödinger equations is obtained. In particular, choosing $C_{1}=C_{2}=C_{3}=C_{4}=0, C_{0}=1$ yields the harmonic oscillator Schrödinger equation (14).

Now let us pass from particular examples to the general case in order to examine which constraints are imposed on the coefficients of the operator (77) by the requirement of conditional invariance (8). In order to compute the commutator on the left-hand side of (8) we use the following identity:

$$
\left[\partial_{x}^{k}, f(x)\right]=\sum_{j=0}^{k-1} \mathrm{C}_{k}^{j} f^{(k-j)}(x) \partial_{x}^{j}, \quad k \in \mathbf{N},
$$

where $\mathrm{C}_{j}^{k}=k !(j !(k-j) !)^{-1}$ are binomial coefficients, which is established by the mathematical induction method with the help of the evident identity

$$
\left[\partial_{x}^{k+1}, f(x)\right] \equiv \partial_{x}\left[\partial_{x}^{k}, f(x)\right]+f^{\prime}(x) \partial_{x}^{k} .
$$

Taking into account formula (19) we rewrite relation (8) as follows:

$$
\begin{aligned}
& -\sum_{j=0}^{n-1} \mathrm{C}_{n}^{j} V^{(n-j)} \partial_{x}^{j}-\sum_{i=1}^{n-1} \sum_{j=0}^{i-1} q_{i} \mathrm{C}_{i}^{j} V^{(i-j)} \partial_{x}^{j}-\sum_{j=0}^{n-1}\left(2 q_{j}^{\prime} \partial_{x}+q_{j}^{\prime \prime}\right) \partial_{x}^{j} \\
& =r(x)\left(\partial_{x}^{n}+\sum_{j=0}^{n-1} q_{j} \partial_{x}^{j}\right) .
\end{aligned}
$$

Comparing the coefficients of $\partial_{x}^{n}$ on the left- and right-hand sides of the above equation we conclude that $r(x)=-2 q_{n-1}^{\prime}$. Comparing the coefficients of the linearly independent operators $\partial_{x}, \partial_{x}^{2}, \ldots, \partial_{x}^{n-1}$ we arrive at the following system of nonlinear ordinary differential equations for the functions $q_{0}(x), q_{1}(x), \ldots, q_{n-1}(x), V(x)$ :

$$
2 q_{j-1}^{\prime}+q_{j}^{\prime \prime}+\mathrm{C}_{n}^{j} V^{(n-j)}+\sum_{i=j+1}^{n-1} q_{i} \mathrm{C}_{i}^{j} V^{(i-j)}-2 q_{n-1}^{\prime} q_{j}=0,
$$

where $j=0,1, \ldots, n-1$ and by convention $q_{-1} \stackrel{\text { def }}{=} 0, q_{n} \stackrel{\text { def }}{=} 1$.

Thus, we have $n$ equations for $n+1$ functions, which means that the system (20) is under-determined. As an immediate consequence of this fact we conclude that any Schrödinger equation (1) is reducible. Indeed, fixing 
in an arbitrary way a function $V=V(x)$ yields a second-order system of $n$ ordinary differential equations for $n$ functions $q_{0}(x), q_{1}(x), \ldots, q_{n-1}(x)$. Each solution of such a system gives rise to an operator $Q$ satisfying by construction condition (8). Consequently, the conditions of Theorem 1 can be fulfilled with any choice of the potential $V(x)$.

Let us demonstrate how the results obtained can be used to study the spectral properties of the Schrödinger equation. Remarkably, to this end we do not need an explicit form of solution of the system of nonlinear ordinary differential equations (20). It suffices to know initial values of the functions $q_{j}(x)$ and of their first derivatives $q_{j}^{\prime}(x)$ at some point $x=x_{0} \in \mathbf{R}$. We denote these as follows

$$
q_{j}\left(x_{0}\right)=A_{j+1}, \quad q_{j}^{\prime}\left(x_{0}\right)=B_{j+1}, \quad j=0,1, \ldots, n-1 .
$$

All the information about spectral properties of the Schrödinger operator restricted to an invariant space $\mathcal{V}_{n}$ with basis elements $u_{1}(x), u_{2}(x), \ldots, u_{n}(x)$ is contained in the matrix $\left\|\Lambda_{j k}\right\|$, which determines a transformation law (2) for the functions $u_{j}(x)$ with respect to the action of the Schrödinger operator $S$.

Let $Q$ be a differential operator of the order $n$ satisfying condition (8) with some choice of the function $V(x)$. Then, by force of Theorem 1, an invariant space $\mathcal{V}_{n}$ of the corresponding Schrödinger operator $S$ is spanned by the fundamental system of solutions of the $n$-th order ordinary differential equation

$$
u^{(n)}(x)+\sum_{j=0}^{n-1} q_{j}(x) u^{(j)}(x)=0 .
$$

We denote this system as $\left\{u_{1}(x), u_{2}(x), \ldots, u_{n}(x)\right\}$. As any $n$ linearly independent solutions of equation (22) form a fundamental system of solutions, there is a freedom in the choice of the functions $u_{j}(x)$. We fix these by imposing initial conditions.

Let $\left\|L_{j k}\right\|$ be a constant non-singular $n \times n$ matrix. Consider the following $n$ Cauchy problems

$$
\begin{aligned}
& u_{k}^{(n)}(x)+\sum_{j=0}^{n-1} q_{j}(x) u_{k}^{(j)}(x)=0, \quad k=1, \ldots, n \\
& u_{k}^{(j-1)}\left(x_{0}\right)=L_{k j}, \quad k, j=1, \ldots, n .
\end{aligned}
$$


It is well-known from the general theory of linear differential equations that the above system has a unique solution, and what is more, this solution yields a fundamental system of solutions of equation (22).

Differentiating relations (2) $n-1$ times with respect to $x$ and excluding the $n$-th and the $(n+1)$-th derivatives of the functions $u_{j}(x)$ with the help of equations (23) we arrive at the following relations:

$$
\begin{aligned}
\sum_{k=1}^{n} \Lambda_{j k} u_{k}^{(i)} & =u_{j}^{(i+2)}-\sum_{k=0}^{i} \mathrm{C}_{i}^{k} V^{(i-k)} u_{j}^{(k)}, \\
\sum_{k=1}^{n} \Lambda_{j k} u_{k}^{(n-2)} & =-\sum_{k=0}^{n-1} q_{k} u_{j}^{(k)}-\sum_{k=0}^{n-2} \mathrm{C}_{n-2}^{k} V^{(n-k-2)} u_{j}^{(k)}, \\
\sum_{k=1}^{n} \Lambda_{j k} u_{k}^{(n-1)} & =\sum_{k=0}^{n-1}\left(q_{n-1} q_{k}-q_{k}^{\prime}-q_{k-1}-\mathrm{C}_{n-1}^{k} V^{(n-k-1)}\right) u_{j}^{(k)},
\end{aligned}
$$

where $j=1, \ldots, n, i=0, \ldots, n-3$ and as above $q_{-1} \stackrel{\text { def }}{=} 0$.

Choosing $x=x_{0}$ in (24) yields

$$
\sum_{k=1}^{n} \Lambda_{j k} L_{k i}=\sum_{k=1}^{n} L_{j k} R_{k i}, \quad j, i=1, \ldots, n,
$$

where

$$
\begin{aligned}
R_{k i} & =\delta_{k i+2}-\mathrm{C}_{i-1}^{k-1} V^{(i-k)}\left(x_{0}\right), \quad i=1, \ldots, n-2, \\
R_{k n-1} & =-A_{k}-\mathrm{C}_{n-2}^{k-1} V^{(n-k-1)}\left(x_{0}\right), \\
R_{k n} & =A_{n} A_{k}-B_{k}-A_{k-1}-\mathrm{C}_{n-1}^{k-1} V^{(n-k)}\left(x_{0}\right) .
\end{aligned}
$$

In formulae (25) the index $k$ runs from 1 to $n, A_{k}, B_{k}$ are constants defined by (21) and $\delta_{k j}$ is the Kronecker symbol.

Rewriting the formulae obtained in the matrix form we have $\Lambda L=L R$, where $\Lambda, L, R$ are constant $n \times n$ matrices with entries $\Lambda_{j k}, L_{j k}, R_{j k}$, respectively. Hence, we derive the explicit form of the matrix $\Lambda$

$$
\Lambda=L R L^{-1} .
$$

Thus we have proved the following assertion. 
Theorem 3 Let $L$ be an arbitrary invertible $n \times n$ matrix, and $A_{1}, \ldots, A_{n}$, $B_{1}, \ldots, B_{n}$ be arbitrary constants. Then, for any choice of the function $V(x)$

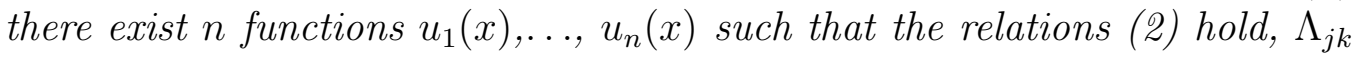
being the entries of the $n \times n$ matrix given by formulae (25), (26).

The above theorem has as a consequence the following important assertion which describes a finite part of the spectrum of the Schrödinger operator $S$.

Theorem 4 Let $\lambda_{1}, \ldots, \lambda_{m}$ be distinct eigenvalues of the matrix $\left\|R_{j k}\right\|$ determined by formulae (25). Then, for any choice of parameters $A_{j}, B_{j}$ there exist linearly independent functions $\psi_{1}(x), \ldots, \psi_{m}(x)$ satisfying the Schrödinger equation (1) with $\varepsilon=\lambda_{1}, \varepsilon=\lambda_{2}, \ldots, \varepsilon=\lambda_{m}$, correspondingly.

The proof follows from Theorem 2 if one takes into account that the matrix $\Lambda$ is similar to $R$ and, consequently, has the same eigenvalues $\lambda_{1}, \lambda_{2}$, $\ldots, \lambda_{m}$. The explicit form of the functions $\psi_{1}(x), \ldots, \psi_{m}(x)$ is given by the formula (凷), where $\vec{a}^{j}=\left(a_{1}^{j}, a_{2}^{j}, \ldots, a_{n}^{j}\right), j=1, \ldots, m$ are eigenvectors of the matrix $\Lambda$ corresponding to the eigenvalues $\lambda_{1}, \lambda_{2}, \ldots, \lambda_{m}$.

Thus, using the conditional symmetry approach we were able not only to calculate the spectrum of the Schrödinger operator $S=\partial_{x}^{2}-V(x)$ but also to construct in an explicit form a $2 n$-parameter family of matrix representations of $S$.

As is seen from formulae (25), (26), there is a large freedom in choice of the matrix $\Lambda$. First, it depends on $2 n$ arbitrary constants $A_{j}, B_{j}$, which fix a fundamental system of solutions of ordinary differential equation (22). Consequently, choosing specific constants $A_{j}, B_{j}$ means fixing a representation space $\mathcal{V}_{n}$. Secondly, it contains an arbitrary constant $n \times n$ matrix $L$. The appearance of the matrix $L$ in the definition of $\Lambda$ reflects a freedom in choosing a basis of the representation space $\mathcal{V}_{n}$. Indeed, if $u_{1}(x), \ldots, u_{n}(x)$ is a basis of the space $\mathcal{V}_{n}$, then the functions $\sum_{k=1}^{n} L_{j k} u_{k}(x)$ also form a basis with an arbitrary invertible constant $n \times n$ matrix $\left\|L_{j k}\right\|$. That is why, choosing a specific matrix $L$ results in fixing a basis of the representation space $u_{1}(x)$, $\ldots, u_{n}(x)$. This freedom can be used, in particular, to obtain an orthogonal basis for $\mathcal{V}_{n}$ (to this end one should apply the standard Gram-Schmidt orthogonalization procedure).

But representations of the Schrödinger operator in these basises are equivalent, which is readily seen from the formula (26). 
Thus, it is established that any Schrödinger equation has $n$-dimensional invariant spaces $\mathcal{V}_{n}$ with arbitrary $n \in \mathbf{N}$. Furthermore, we have constructed the $2 n$-parameter family of matrix representations of the corresponding Schrödinger operator in these spaces (the formulae (25), (26)). But to obtain an explicit form of the basis of $\mathcal{V}_{n}$ we still have

- to integrate system of nonlinear ordinary differential equations (20), and

- to construct the general solution of the n-th order ordinary differential equation (22).

We will demonstrate that using a simple trick we may avoid the necessity to integrate equation (22). The said trick is based on the fact that we need not all solutions of (22) but only those which simultaneously satisfy the initial Schrödinger equation (11). This means that we have to solve the following over-determined system of two ordinary differential equations:

$$
\left\{\begin{array}{l}
\psi_{x x}=(\varepsilon+V(x)) \psi \\
\psi^{(n)}(x)+\sum_{j=0}^{n-1} q_{j}(x) \psi^{(j)}(x)=0 .
\end{array}\right.
$$

Using the first equation and its differential consequences up to the order $n-2$ we can exclude from the second equation all the derivatives of the function $\psi$ of the order $j>1$ and rewrite system (27) in the following equivalent form:

$$
\left\{\begin{array}{l}
\psi_{x x}=(\varepsilon+V(x)) \psi, \\
\left(\sum_{i=0}^{N} a_{i}(x) \varepsilon^{i} \partial_{x}+\sum_{i=0}^{N} b_{i}(x) \varepsilon^{i}\right) \psi=0,
\end{array}\right.
$$

where $N=\left[\frac{n}{2}\right], a_{i}(x), b_{i}(x)$ are linear combinations of the functions $q_{j}(x)$ with coefficients depending on $V(x)$ and its derivatives (and, consequently, independent of $\varepsilon$ ) and, furthermore, $a_{N}=1$ if $n=2 N+1$ and $a_{N}=0, b_{N}=1$ if $n=2 N$.

The compatibility condition for the above system reads as

$$
\left(\frac{\sum_{i=0}^{N} b_{i}(x) \varepsilon^{i}}{\sum_{i=0}^{N} a_{i}(x) \varepsilon^{i}}\right)_{x}-\left(\frac{\sum_{i=0}^{N} b_{i}(x) \varepsilon^{i}}{\sum_{i=0}^{N} a_{i}(x) \varepsilon^{i}}\right)^{2}+V(x)+\varepsilon=0 .
$$


As functions $a_{i}, b_{i}$ are independent of $\varepsilon$, coefficients of the powers of $\varepsilon$ should be independent of $x$. This requirement yields

1) $2 N+1$ ordinary differential equations

$$
\sum_{i+j=k}\left(b_{i}^{\prime} a_{j}-b_{i} a_{j}^{\prime}-b_{i} b_{j}+V a_{i} a_{j}\right)+\sum_{i+j=k-1} a_{i} a_{j}=C_{k}
$$

where $k=0,1, \ldots, 2 N$, for $2 N+2$ functions $a_{0}, \ldots, a_{N-1}, b_{0}, \ldots, b_{N}$, $V$, provided $n=2 N+1$, or

2) $2 N$ ordinary differential equations of the form (30) with $k$ taking the values $0,1, \ldots, 2 N-1$ for $2 N+1$ functions $a_{0}, \ldots, a_{N-1}, b_{0}, \ldots, b_{N-1}$, $V$, provided $n=2 N$.

In (30) $C_{j}$ are arbitrary constants.

Provided conditions (30) are fulfilled, the compatibility condition (29) reduces to an algebraic equation

1) under $n=2 N+1$

$$
\varepsilon^{2 N+1}+\sum_{j=0}^{2 N} C_{j} \varepsilon^{k}=0
$$

2) under $n=2 N$

$$
\varepsilon^{2 N}+\sum_{j=0}^{2 N-1} C_{j} \varepsilon^{k}=0
$$

and the general solution of system (28) is given by the quadrature

$$
\psi(x)=\exp \left\{-\int\left(\frac{\sum_{i=0}^{N} b_{i}(x) \varepsilon^{i}}{\sum_{i=0}^{N} a_{i}(x) \varepsilon^{i}}\right) d x\right\} .
$$

It should be noted that equations (31), (32) are nothing else but characteristic equations for the matrix $R$ defined by (25). Their solutions $\lambda_{1}, \ldots, \lambda_{m}$ are eigenvalues and $\psi_{1}(x)=\left.\psi(x)\right|_{\varepsilon=\lambda_{1}}, \ldots, \psi_{m}(x)=\left.\psi(x)\right|_{\varepsilon=\lambda_{m}}$ are eigenfunctions of the corresponding Schrödinger operator $S=\partial_{x}^{2}-V(x)$. 


\section{Symmetry and exact solvability}

With the best of our knowledge the first paper, where a systematic study of high order Lie symmetries of the Schrödinger equations with non-vanishing potentials has been undertaken is the one by Nikitin, ONUFRIYCHUK and FUSHCHYCH [31]. In particular, for several one-dimensional exactly solvable models third-order symmetry operators were constructed. Furthermore, it was conjectured that exact integrability of the Schrödinger equation (1) is intimately connected with its symmetry properties. This conjecture has been confirmed in [24], where a number of exactly solvable potentials were obtained by means of third-order symmetries of (11) and, furthermore, a method for integrating the corresponding Schrödinger equations was suggested. Using a technique developed in the previous section we will demonstrate how to derive exact integrability of equation (四) from its symmetry properties in the class of arbitrary order symmetry operators.

The $n$-th order operator $Q$ of the form (5) is a symmetry operator of equation (1) if the condition (2) is satisfied with $r(x)=0$. As coefficients of $Q$ are independent of $\varepsilon$, equality (2) is only possible when $P \equiv 0$. Computing the commutator on the left-hand side of (2) with $r=0, P=0$ and equating to zero the coefficients of $\partial_{x}^{n+1}$ and $\partial_{x}^{n}$ we conclude that $q_{n}(x)=$ const, $q_{n-1}(x)=$ const. Consequently, any $n$-th order symmetry operator admitted by the Schrödinger equation (11) can be represented in the form (5) with $q_{n}=C=$ const $\neq 0, q_{n-1}=C_{0}=$ const.

First, following 24] we will consider in more detail the third-order symmetry operators admitted by the Schrödinger equation (1). These are the lowest order symmetry operators not equivalent to usual first-order Lie symmetries. The general form of a third-order symmetry operator is as follows (we choose $C=1$ )

$$
Q=\partial_{x}^{3}+C_{0} \partial_{x}^{2}+q_{1}(x) \partial_{x}+q_{0}(x)
$$

where $q_{0}(x), q_{1}(x)$ are sufficiently smooth functions to be determined from the invariance condition (2) with $n=3, r=P=0$. A short computation yields the following expressions for the coefficients of the operator $Q$

$$
q_{0}(x)=C_{2}-C_{0} V(x)-\frac{3}{4} V^{\prime}(x), \quad q_{1}(x)=C_{1}-\frac{3}{2} V(x),
$$


where $V(x)$ is an arbitrary solution of the third-order nonlinear ordinary differential equation

$$
-4 C_{1} V^{\prime}+6 V V^{\prime}-V^{(3)}=0 .
$$

Integrating twice the above equation we arrive at the first-order ordinary differential equation integrable in elliptic functions

$$
C_{4}+2 C_{3} V(x)+4 C_{1} V(x)^{2}-2 V(x)^{3}+V^{\prime}(x)^{2}=0 .
$$

It was established in 24 that particular cases of almost all exactly solvable potentials which can be expressed in elementary functions, such as the trigonometric and hyperbolic Pöschel-Teller, Eckart, Kratzer potentials, potential well of finite and infinite depth, are obtained as solutions of the equation (36).

This fact imply an existence of an intimate connection between high order Lie symmetries and exact solvability of the Schrödinger equations. In what follows we will show that this is not simply a conjecture but a fundamental fact making it possible to classify exactly solvable models and to construct their exact solutions in an explicit form (see, also [24, 25]).

It is straightforward to check that if $Q$ is a Lie symmetry of the Schrödinger equation (1), then $\tilde{Q}=Q+P\left(\partial_{x}^{2}-V(x)-\varepsilon\right)$, where $P$ is an arbitrary differential operator, is also its Lie symmetry. Furthermore, systems of ordinary differential equations

$$
\left(\partial_{x}^{2}-V(x)-\varepsilon\right) \psi(x)=0, \quad Q \psi(x)=0
$$

and

$$
\left(\partial_{x}^{2}-V(x)-\varepsilon\right) \psi(x)=0, \quad \tilde{Q} \psi(x)=0
$$

are equivalent in a sense that they have the same solutions. Making use of these facts we can reduce the $n$-th order symmetry operator $Q$ to a first-order symmetry operator, the coefficients of which are $N$-th order polynomials in $\varepsilon$ (we will preserve the same designation $Q$ for the reduced operator)

$$
Q=a(x, \varepsilon) \partial_{x}+b(x, \varepsilon) \equiv\left(\sum_{j=0}^{N} a_{j}(x) \varepsilon^{j}\right) \partial_{x}+\sum_{j=0}^{N} b_{j}(x) \varepsilon^{j} .
$$

From the invariance condition for the reduced operator

$$
\left[\partial_{x}^{2}-V(x)-\varepsilon, a(x, \varepsilon) \partial_{x}+b(x, \varepsilon)\right]=R(x, \varepsilon)\left(\partial_{x}^{2}-V(x)-\varepsilon\right)
$$


we get a system of determining equations for the coefficients of $Q$

$$
\begin{aligned}
& a^{\prime \prime}(x, \varepsilon)+2 b^{\prime}(x, \varepsilon)=0, \\
& b^{\prime \prime}(x, \varepsilon)+a(x, \varepsilon) V^{\prime}(x)+2 a^{\prime}(x, \varepsilon)(V(x)+\varepsilon)=0,
\end{aligned}
$$

where primes denote differentiation with respect to $x$.

Splitting the above equations by powers of $\varepsilon$ with subsequent integrating yields

$$
\begin{aligned}
& b_{j}(x)=-\frac{1}{2} a_{j}^{\prime}(x)+B_{j}, \quad a_{N}(x)=A_{N}, \\
& a_{j-1}(x)=\frac{1}{4} a_{j}^{\prime \prime}(x)-V(x) a_{j}(x)+\frac{1}{2} \int V^{\prime}(x) a_{j}(x) d x+A_{j-1} .
\end{aligned}
$$

In (38) $A_{-1}, A_{j}, B_{j}$ are arbitrary constants, $j=0,1, \ldots, N, a_{-1}(x) \stackrel{\text { def }}{=} 0$.

Thus, the problem of describing $n$-th order symmetry operators of the Schrödinger equation is reduced to solving the recurrent relations

$$
a_{j-1}(x)=\frac{1}{4} a_{j}^{\prime \prime}(x)-V(x) a_{j}(x)+\frac{1}{2} \int V^{\prime}(x) a_{j}(x) d x+A_{j-1}
$$

with $a_{N}(x)=A_{N}=$ const, $a_{-1}(x) \stackrel{\text { def }}{=} 0, j=N, N-1, \ldots, 0$.

The first $N$ relations $(j=N, N-1, \ldots, 1)$ are solved by subsequent integrations yielding the expressions for the functions $a_{0}(x), \ldots, a_{N-1}(x)$ via the function $V(x)$ and its derivatives. Substituting these results into the last equation $(j=0)$ we arrive at the $2 N$-th order nonlinear ordinary differential equation for the function $V(x)$. It will be shown that any solution of this equation gives rise to an exactly solvable Schrödinger equation (11).

To reveal the structure of the equation in question we introduce the new functions $U_{0}(x), U_{1}(x), \ldots$ by the following recurrence relation:

$$
U_{j}(x)=X U_{j-1}(x) \equiv\left(\frac{1}{4} \partial_{x}^{2}-V(x)+\frac{1}{2} \partial_{x}^{-1} V^{\prime}(x)\right) U_{j-1}, \quad j=0,1, \ldots
$$

where $\partial_{x}^{-1}$ denotes integration with respect to $x$ and $U_{-1}(x) \stackrel{\text { def }}{=} 1$.

Formally, a definition of functions $U_{j}(x)$ contains a quadrature but integrating relations (40) successively we can get rid of it for any $j=1,2,3, \ldots$. Below, we adduce expressions of the functions $U_{j}(x)$ for $j=0,1,2,3$.

$$
U_{0}(x)=-\frac{1}{2} V(x)
$$




$$
\begin{aligned}
U_{1}(x)= & \frac{1}{2^{3}}\left(3 V(x)^{2}-V^{\prime \prime}(x)\right) \\
U_{2}(x)= & \frac{1}{2^{5}}\left(-10 V(x)^{3}+5 V^{\prime}(x)^{2}+10 V(x) V^{\prime \prime}(x)-V^{(4)}(x)\right) \\
U_{3}(x)=\frac{1}{2^{7}}( & 35 V(x)^{4}-70 V(x) V^{\prime}(x)^{2}-70 V(x)^{2} V^{\prime \prime}(x)+21 V^{\prime \prime}(x)^{2} \\
& \left.+28 V^{\prime}(x) V^{(3)}(x)+14 V(x) V^{(4)}(x)-V^{(6)}(x)\right) .
\end{aligned}
$$

Now we can solve the first $N$ relations of (39) in terms of the functions $U_{j}(x)$

$$
a_{N-j}(x)=\sum_{k=0}^{j-1} A_{N-k} U_{j-k-1}(x)+A_{N-j}, \quad j=1, \ldots, N .
$$

Inserting the above expressions into the last equation of (39) we get

$$
A_{-1}+\sum_{k=0}^{N} A_{N-k} U_{N-k}(x)=0
$$

(when deriving the above equation we take into account that by convention $\left.a_{-1}(x)=0\right)$.

Equation (42) is the necessary and sufficient condition for the Schrödinger equation (1) to be invariant with respect to the first-order operator (37), whose coefficients are polynomials in $\varepsilon$ of the order $N$. But it is easy to see that given a condition (42), equation (11) admits operator of the form (37), whose coefficients are polynomials in $\varepsilon$ of an arbitrary order $N^{\prime}>N$.

Indeed, the invariance conditions for the operator

$$
Q=\left(\sum_{j=0}^{N^{\prime}} a_{j}(x) \varepsilon^{j}\right) \partial_{x}+\sum_{j=0}^{N^{\prime}} b_{j}(x) \varepsilon^{j}
$$

have the form (38) with $N=N^{\prime}$. Coefficients $a_{j}(x)$ with $j=N^{\prime}, N^{\prime}-$ $1, \ldots, N^{\prime}-N$ are given by formulae (41), where one should replace $N$ by $N^{\prime}$, and the remaining coefficients by force of relation (42) read as

$$
a_{j}(x)=\sum_{k=0}^{N-1} \tilde{A}_{j k} U_{k}(x)+A_{j}, \quad j=0,1, \ldots, N^{\prime}-N-1,
$$

where $A_{0}, \ldots, A_{N^{\prime}-N-1}$ are arbitrary constants, $\tilde{A}_{j k}$ are constants expressed via $A_{N^{\prime}-N}, \ldots, A_{N^{\prime}}$. 
Substituting these results into the last $(j=0)$ equation from (41) yields

$$
A_{-1}+A_{0} U_{0}(x)+\sum_{k=0}^{N-1} \tilde{A}_{0 k} U_{k+1}(x)=0,
$$

whence we conclude that, provided $A_{-1}=A_{0}=0, \tilde{A}_{0 k}=0, k=0,1, \ldots, N-$ 1 , the invariance conditions are satisfied.

Thus, if equation (12) is fulfilled with some $N \in \mathbf{N}$, then the corresponding Schrödinger equation admits arbitrary order Lie symmetries and, consequently, is exactly solvable.

A general solution of the Schrödinger equation invariant under the operator $Q$ is given by (33). Substituting formula (33) into equation (11), where $V(x)$ is an arbitrary solution of (42), results in a $(2 N+1)$-th order algebraic equation for $\varepsilon$. Its solutions $\varepsilon=\lambda_{1}, \ldots, \varepsilon=\lambda_{m}$ are eigenvalues of the Schrödinger operator. Corresponding eigenfunctions are obtained if we insert $\varepsilon=\lambda_{1}, \ldots, \varepsilon=\lambda_{m}$ into (33).

Summing up, we conclude that any solution $V(x)$ of (42) gives rise to an exactly solvable Schrödinger equation. In what follows it will be established that the more strong assertion holds. Namely, if $V(x)$ is a solution of (42) with some $N$, then the corresponding Schrödinger equation may have an arbitrary spectrum.

Theorem 5 Let $V=V(x)$ be a solution of ordinary differential equation (49) with some fixed $N \in \mathbf{N}$. Then, the Schrödinger equation (1) is exactly solvable and, moreover, the Schrödinger operator $S=\partial_{x}^{2}-V(x)$ may have an arbitrarily prescribed spectrum.

Proof. We will give the principal steps of the proof omitting technical details. As the potential $V(x)$ satisfies equation (42), the corresponding Schrödinger equation admits a Lie symmetry of the form (37). Excluding from (37) the parameter $\varepsilon$ we recover symmetry operator $Q$ of the order $n=2 N+1$ which commutes with the Schrödinger operator $S$. Next, we construct an operator $Q_{1}=Q+f(\varepsilon)$, where $f(\varepsilon)$ is an arbitrary smooth function. Evidently, $Q_{1}$ commutes with $S$ and, consequently, is a symmetry operator of the Schrödinger equation. This means that conditions of Theorem 1 are fulfilled and a fundamental system of solutions of ordinary differential equation

$$
Q_{1} u(x)=0
$$


forms a basis of the invariant space $\mathcal{V}_{n}$ of the corresponding Schrödinger operator $S$. Representation of $S$ in the space $\mathcal{V}_{n}$ is given by the formulae (25), (26), where $A_{1}=a+f(\varepsilon)$ and parameters $a, A_{2}, \ldots, A_{n}, B_{1}, \ldots, B_{N}$ are independent of $f(\varepsilon)$. Eigenvalues of the operator $S$ are solutions of the characteristic equation for the matrix $R$ having the entries (25), i.e. of the equation

$$
\operatorname{det}\left\|R_{j k}-\varepsilon \delta_{j k}\right\|=0
$$

It is not difficult to become convinced of that the above equation can be represented in the form

$$
p_{0}(\varepsilon)+p_{1}(\varepsilon) f(\varepsilon)+p_{2}(\varepsilon) f(\varepsilon)^{2}=0,
$$

where $p_{0}, p_{1}, p_{2}$ are polynomials in $\varepsilon$ of the order not higher than $n$.

Consequently, zeros of the function

$$
F(\varepsilon)=p_{0}(\varepsilon)+p_{1}(\varepsilon) f(\varepsilon)+p_{2}(\varepsilon) f(\varepsilon)^{2}
$$

are eigenvalues of the Schrödinger operator.

As $f(\varepsilon)$ is arbitrary, the function $F(\varepsilon)$ may have an arbitrarily prescribed set of zeros and, thus, a spectrum of the initial Schrödinger equation (11) may be arbitrary.

As an illustration, we will consider the simplest case when there exists such $N_{1}$ that $U_{N_{1}}(x)=0$. In such a case, the coefficients of the symmetry operator (37) with $N=k N_{1}, k \in \mathbf{N}$ are easily shown to be

$$
\begin{aligned}
& a(x, \varepsilon)=\left(\sum_{j=-1}^{N_{1}-1} U_{j}(x) \varepsilon^{N_{1}-j-1}\right)\left(\sum_{j=0}^{k} A_{j} \varepsilon^{j}\right) \\
& b(x, \varepsilon)=-\frac{1}{2}\left(\sum_{j=-1}^{N_{1}-1} U_{j}^{\prime}(x) \varepsilon^{N_{1}-j-1}\right)\left(\sum_{j=0}^{k} A_{j} \varepsilon^{j}\right)+\left(\sum_{j=0}^{N} B_{j} \varepsilon^{j}\right)
\end{aligned}
$$

where $A_{0}, \ldots, A_{k}, B_{0}, \ldots, B_{N}$ are arbitrary constants.

Inserting this result into (41) and integrating we come to the following Ansatz for the function $\psi(x)$ :

$$
\begin{aligned}
\psi(x)= & \left(\sum_{j=-1}^{N_{1}-1} U_{j}(x) \varepsilon^{N_{1}-j-1}\right)^{\frac{1}{2}} \\
& \times \exp \left\{-f(\varepsilon) \int \frac{d x}{\sum_{j=-1}^{N_{1}-1} U_{j}(x) \varepsilon^{N_{1}-j-1}}\right\},
\end{aligned}
$$


where $f(\varepsilon)=\left(\sum_{j=0}^{N} B_{j} \varepsilon^{j}\right)\left(\sum_{j=0}^{k} A_{j} \varepsilon^{j}\right)^{-1}$.

Substitution of the expression (43) into the initial equation after some manipulations gives the following algebraic equation:

$$
\varepsilon^{2 N_{1}+1}+\sum_{j=0}^{N_{1}-1} \mathcal{I}_{j} \varepsilon^{j}-f(\varepsilon)^{2}=0
$$

where $\mathcal{I}_{j}$ are integrals of the ordinary differential equation $U_{N_{1}}(x)=0$ and, consequently, are constant on the set of its solutions.

Thus, for an arbitrary $N_{1} \in \mathbf{N}$ any solution $V=V(x)$ of the ordinary differential equation $U_{N_{1}}(x)=0$ gives rise to an exactly solvable Schrödinger equation. Eigenvalues of the Schrödinger operator $S$ are the roots of the algebraic equation (44) and the eigenfunctions are of the form (43), where $\varepsilon$ is an arbitrary solution of (44).

It is instructive to consider in more detail the above formulae for the case $N=N_{1}=1$. With this choice of $N$ the formula (43) reads as

$$
\psi(x)=(2 \varepsilon-V(x))^{\frac{1}{2}} \exp \left\{-2 f(\varepsilon)\left(\int \frac{d x}{2 \varepsilon-V(x)}\right)\right\},
$$

where $f(\varepsilon)=\left(A_{1} \varepsilon+A_{0}\right)\left(B_{1} \varepsilon+B_{0}\right)^{-1}$ and the function $V(x)$ is a solution of the ordinary differential equation $U_{1}(x)=0$, i.e.

$$
-3 V(x)^{2}+V^{\prime \prime}(x)=0 .
$$

Inserting the Ansatz (45) into (11) yields the following equality:

$$
-8 \varepsilon^{3}+\mathcal{I}_{0}+8 f(\varepsilon)^{2}=0,
$$

where

$$
\mathcal{I}_{0}=V(x)^{3}-\frac{1}{2} V^{\prime}(x)^{2}
$$

is the first integral of equation (46). Note that an alternative derivation of the formulae (45)-(47) has been obtained in [25].

Now let us make an important remark. It is readily seen from formulae (45) - (47) that the function $f(\varepsilon)$ is not obliged to be a ratio of two firstorder polynomials. It may be arbitrary. And what is more, eigenvalues of the Schrödinger operator are the zeros of the function $F(\varepsilon)=-8 \varepsilon^{3}+\mathcal{I}_{0}+$ 
$8 f(\varepsilon)^{2}$. Choosing an arbitrary function $f(\varepsilon)$ in a proper way we can get the function $F(\varepsilon)$ having arbitrary prescribed set of zeros. This means that the Schrödinger equation (1) with $V(x)$ satisfying (47) may have an arbitrary spectrum.

For example, if we choose $f=\sqrt{\varepsilon^{3}-\mathcal{I}_{0} / 8}$, then the function (45) is a solution of the Schrödinger equation under arbitrary $\varepsilon$ (the case of a continuous spectrum). Next, if we choose $f=\sqrt{\varepsilon^{3}-\mathcal{I}_{0} / 8+\sum_{j=0}^{N} A_{j} \varepsilon^{j}}$, then a finite discrete spectrum is obtained (eigenvalues are roots of the $N$-th order polynomial). At last, choosing $f=\sqrt{\varepsilon^{3}-\mathcal{I}_{0} / 8+\sin \varepsilon}$ yields an infinite discrete spectrum (eigenvalues are zeros of the $\sin \varepsilon$ ).

Similar results are obtained for $N=N_{1}=2$

$$
\begin{aligned}
\psi(x)= & \left(8 \varepsilon^{2}-4 \varepsilon V(x)+3 V(x)^{2}-V^{\prime \prime}(x)\right)^{\frac{1}{2}} \\
& \times \exp \left\{-8 f(\varepsilon)\left(\int \frac{d x}{8 \varepsilon^{2}-4 \varepsilon V(x)+3 V(x)^{2}-V^{\prime \prime}(x)}\right)\right\},
\end{aligned}
$$

where $f(\varepsilon)$ is an arbitrary function, $V(x)$ is a solution of the ordinary differential equation

$$
10 V(x)^{3}-5 V^{\prime}(x)^{2}-10 V(x) V^{\prime \prime}(x)+V^{(4)}(x)=0
$$

and $\varepsilon$ is a solution of the equation

$$
-128 \varepsilon^{5}-2 \varepsilon \mathcal{I}_{1}+\mathcal{I}_{0}+128 f(\varepsilon)^{2}=0 .
$$

Here $\mathcal{I}_{0}, \mathcal{I}_{1}$ are integrals of equation (48) of the form

$$
\begin{aligned}
\mathcal{I}_{0}= & 5 V(x)^{4}-10 V(x) V^{\prime}(x)^{2}-V^{\prime \prime}(x)^{2}+2 V^{\prime}(x) V^{(3)}(x), \\
\mathcal{I}_{1}= & -\frac{\mathcal{I}_{0} V(x)}{2}+\frac{19 V(x)^{5}}{2}-\frac{\mathcal{I}_{0}^{2}}{8 V^{\prime}(x)^{2}}-\frac{5 \mathcal{I}_{0} V(x)^{4}}{4 V^{\prime}(x)^{2}}-\frac{25 V(x)^{8}}{8 V^{\prime}(x)^{2}} \\
& +\frac{5 V(x)^{2} V^{\prime}(x)^{2}}{2}-10 V(x)^{3} V^{\prime \prime}(x)-V^{\prime}(x)^{2} V^{\prime \prime}(x)+\frac{5 V(x) V^{\prime \prime}(x)^{2}}{2} \\
& +\frac{\mathcal{I}_{0} V^{\prime \prime}(x)^{2}}{4 V^{\prime}(x)^{2}}+\frac{5 V(x)^{4} V^{\prime \prime}(x)^{2}}{4 V^{\prime}(x)^{2}}-\frac{V^{\prime \prime}(x)^{4}}{8 V^{\prime}(x)^{2}} .
\end{aligned}
$$

Generically, if $V(x)$ is a solution of the $2 N$-th order ordinary differential equation $U_{N}(x)=0$, then the corresponding Schrödinger operator $S$ 
may have an arbitrary spectrum. Eigenvalues of $S$ are obtained by solving the algebraic equation (44) and its eigenfunctions by substituting the corresponding values for $\varepsilon$ into (43).

We will finish this section with one more puzzling property of the exactly integrable models obtained. Let us denote the total derivatives of the functions $U_{j}(x)$ with respect to $x$ as $W_{j}(x)$ and consider an infinite set of evolution equations for a function $u=u(t, x)$

$$
\frac{\partial u(t, x)}{\partial t}=F_{j}[u(t, x)], \quad j>1
$$

where the functions $F_{j}$ are obtained from $W_{j}(x)$ by formal replacement of $V(x)$ with $u(t, x)$. Now we see that equations obtained in this way form the famous integrable $\mathrm{KdV}$ hierarchy. Taking, for example, $j=2$ yields the KdV equation

$$
\frac{\partial u(t, x)}{\partial t}=\frac{1}{2^{3}}\left(6 u(t, x) \frac{\partial u(t, x)}{\partial x}-\frac{\partial^{3} u(t, x)}{\partial x^{3}}\right) .
$$

Furthermore, differentiating the relations (40) we obtain the recurrence relations determining $W_{j}(x)$

$$
W_{j+1}(x)=Y W_{j}(x) \equiv\left(\frac{1}{4} \partial_{x}^{2}-V(x)-\frac{1}{2} V^{\prime}(x) \partial_{x}^{-1}\right) W_{j}(x), \quad j=0,1, \ldots
$$

with $W_{0}(x)=0$. The operator $Y$ above is nothing else but the well-known recurrence operator for the $\mathrm{KdV}$ hierarchy [32].

Next, if we formally replace $V(x)$ by $u(t, x)$ in $U_{j}(x)$ determined by the recurrence relations (40), then the densities of motion constants of the KdV equation are obtained, $X$ being the recursion operator connecting these densities.

Equations of the form (42) are known in the literature as the stationary Lax-Novikov equations. Equations of the stationary KdV hierarchy are particular cases of equations (42) with $A_{1}=\cdots=A_{N-1}=0$. We have proved that any solution $V=V(x)$ of the stationary Lax-Novikov hierarchy (42) yields an exactly solvable Schrödinger equation. The correspondence between solutions of stationary KdV hierarchy and exactly solvable Schrödinger equations with reflection-less potentials is known (see, e.g. the paper [33] and references therein). Moreover, it has been established that the Schrödinger 
operators with so chosen potentials may have an arbitrarily prescribed spectrum. But the fact that solutions of the stationary Lax-Novikov hierarchy (42) have the same property seems to be new.

\section{Conclusion}

In view of numerous excellent papers and monographs (see e.g. [17] and the literature cited therein) devoted to developing algebraic methods for the investigation of spectral properties of equation (11), it is, of course, not enough to say that the problem of describing part of the spectrum of the Schrödinger equation is equivalent to computing its conditional symmetries in order to justify a necessity of introducing a new complicated structure. Our principal motivation for looking for a symmetry interpretation of the results obtained in this field is that it may open a possibility

- to study the spectrum of two- and three-dimensional Schrödinger equations,

- to investigate 'spectral properties' of nonlinear Scrödinger equations, and

- to study spectral properties of matrix differential operators (say, of the Dirac operator)

by purely algebraic means.

For instance, there are strong evidences that a necessary condition for a three-dimensional Schrödinger equation to be exactly solvable is an invariance with respect to a three-dimensional Lie algebra of high order symmetry operators. We can guess that one of the necessary conditions of 'quasi exact solvability' of the three-dimensional Schrödinger equations is a non-trivial conditional symmetry admitted. The simplest possibility to move in this direction is to combine the technique developed in the present paper with the method of separation of variables [34]. In our paper [35] we have classified potentials $V\left(x_{1}, x_{2}\right)$ such that the corresponding two-dimensional Schrödinger equation

$$
i \psi_{t}+\psi_{x_{1} x_{1}}+\psi_{x_{2} x_{2}}=V\left(x_{1}, x_{2}\right) \psi
$$

can be separated into three ordinary differential equations. One of these is a first-order equation and can always be integrated by quadratures. Two other 
are exactly of the form (1) and can be solved within the framework of the approach described in Sections 2 and 3.

Furthermore, the method of conditional symmetries is applicable not only to linear partial differential differential equations but also to nonlinear ones [21, 22]. Let, for example, the one-dimensional nonlinear Schrödinger equation

$$
\psi_{x x}=\left(\varepsilon+V(x)+F\left(\psi, \psi^{*}, \psi_{x}, \psi_{x}^{*}\right)\right) \psi
$$

be conditionally invariant with respect to an $n$-th order Lie-Bäcklund operator

$$
Q=\eta\left(x, \psi, \psi^{\prime}, \ldots, \psi^{(n)}\right) \partial_{\psi}+\cdots
$$

in the sense of [22]. Then, using a technique similar to that developed in Section 2 we can construct an Ansatz for a function $\psi(x)$, which gives a solution of (50), provided the energy parameter $\varepsilon$ satisfy some algebraic equation $G(\varepsilon)=0$. Solutions of this equation can be interpreted as eigenvalues of the nonlinear Schrödinger operator $\partial_{x}^{2}-V-F$.

An interesting example is a family of nonlinear Schrödinger equations suggested by Doebner \& Goldin [36]. Taking the polar decomposition

$$
\psi(t, x)=\mathrm{e}^{r(t, x)+i s(t, x)},
$$

and fixing the gauge $\nu_{1}=-1, \nu_{2}=0$ (this is always possible [37) we can represent the Doebner-Goldin model in the following way:

$$
\begin{aligned}
& r_{t}+s_{x x}+2 r_{x} s_{x}=0, \\
& s_{t}+2 \mu_{2} r_{x x}+\mu_{1} s_{x x}+4\left(\mu_{2}+\mu_{5}\right) r_{x}^{2} \\
& \quad+2\left(\mu_{1}+\mu_{4}\right) r_{x} s_{x}+\mu_{3} s_{x}^{2}+\mu_{0} V(x)=0,
\end{aligned}
$$

where $\mu_{0}, \ldots, \mu_{5}$ are model parameters.

If we impose on the solutions of (52) an additional condition $s_{x}=0$ (which picks out a subset of stationary solutions), then the system obtained is consistent if and only if the conditions

$$
r=r(x), \quad s=-\varepsilon t, \quad \varepsilon=\text { const } \in \mathbf{R}
$$

are fulfilled.

With this choice of functions $r$ and $s$ system (52) reduces to a single equation

$$
-\varepsilon+2 \mu_{2} r^{\prime \prime}+4\left(\mu_{2}+\mu_{5}\right)\left(r^{\prime}\right)^{2}+\mu_{0} V(x)=0,
$$


which is either linear $\left(\mu_{2}+\mu_{5}=0\right)$ or can be linearized by the substitution

$$
\varphi(x)=\exp \left\{\frac{\mu_{2}}{2\left(\mu_{2}+\mu_{5}\right)} r(x)\right\}
$$

to become

$$
\varphi^{\prime \prime}=\left(\frac{\varepsilon}{4\left(\mu_{2}+\mu_{5}\right)}-\frac{\mu_{0}}{4\left(\mu_{2}+\mu_{5}\right)} V(x)\right) \varphi .
$$

As established in Section 2, any equation of the form (56) possesses a nontrivial high order conditional symmetry. Since the nonlinear equation (54) is equivalent to (56), it possesses high order conditional symmetry as well. Thus, the initial Doebner-Goldin equation has a subset of solutions with non-trivial conditional symmetry which can be effectively applied to construct finite or even infinite (if the conditional symmetry can be reduced to a high order Lie symmetry) set of its exact solutions.

We hope that the reasonings above are convincing enough to motivate a further study of high order conditional symmetries of linear and nonlinear Schrödinger equations in one, two and three dimensions. It may be also very interesting to study classical and conditional symmetries of the stationary Dirac equation in the presence of non-vanishing electro-magnetic field and to apply these to derive a spectrum of the Dirac operator. These problems are under investigation now and will be a topic of our future publications.

\section{Acknowledgments}

The results of in the present paper were obtained by the author when he was staying at the Arnold-Sommerfeld Institute for Mathematical Physics, Technical University of Clausthal as the Alexander von Humboldt Research Fellow. Author wishes to thank the Director of AS Institute H.-D. DoeBNER for hospitality. Special thanks are addressed to W. FushCHYCH and A. Nikitin for critical remarks and suggestions and also to A. UsHVERIDZE, who introduced the author into the exciting world of quasi exactly solvable models. Furthermore, author express his gratitude to P. NATTERmann for critical reading the manuscript and pointing out the possibility of linearization of the one-dimensional Doebner-Goldin model restricted to a subset of stationary solutions. 


\section{References}

[1] W.I. Fushchych, Symmetry and Solutions of Nonlinear Mathematical Physics Equations (Institute of Mathematics, Kiev, 1987), p.4.

[2] W.I. Fushchych, Ukrain. Math. J., 39, 116 (1987).

[3] W.I. Fushchych and A.G. Nikitin, Symmetries of Maxwell's Equations (Reidel, Dordrecht, 1987).

[4] W.I. Fushchych, W.M. Shtelen, and N.I. Serov, Symmetry Analysis and Exact Solutions of Nonlinear Equations of Mathematical Physics (Kluwer Academic Publishers, Dordrecht, 1993).

[5] W.I. Fushchych, Proceedings of the International Workshop "Modern Group Analysis" (Kluwer Academic Publishers, Dordrecht, 1993), p.231.

[6] P. Clarkson and M. Kruskal, J. Math. Phys., 30, 2201 (1989).

[7] P. Olver and Ph. Rosenau, Phys. Lett. A, 112, 107 (1987).

[8] Levi D. and Winternitz P., J. Phys. A: Math. Gen., 22, 2915 (1989).

[9] W.I. Fushchych and R.Z. Zhdanov, Phys. Rep., 172, 123 (1989).

[10] W.I. Fushchych and R.Z. Zhdanov, J. Math. Phys., 32, 3488 (1991).

[11] W.I. Fushchych, R.Z. Zhdanov, and I.V.Revenko, Ukrain. Math. J., 43, 1471 (1991).

[12] R.Z. Zhdanov, I.V. Revenko and W.I. Fushchych, J. Math. Phys., 36, 7109 (1995).

[13] R.Z. Zhdanov and W.I. Fushchych, J. Phys. A: Math. Gen., 28, 6253 (1995).

[14] G.W. Bluman and J.D. Cole, J. Math. Mech., 18, 1025 (1969).

[15] A.V. Turbiner, Comm. Math. Phys., 118, 467 (1988). 
[16] M.A. Shifman and A.V. Turbiner, Comm. Math. Phys., 126, 347 (1989).

[17] A.G. Ushveridze, Quasi-exactly solvable models in quantum mechanics (IOP Publ., Bristol, 1993).

[18] P. Olver, Applications of Lie Groups to Differential Equations (Springer, New York, 1986).

[19] L.V. Ovsjannikov, Group Properties of Differential Equations (Nauka, Novosibirsk, 1962).

[20] W.I. Fushchych and R.Z. Zhdanov, Ukrain. Math. J., 44, 970 (1992).

[21] W.I. Fushchych and R.Z. Zhdanov, Proc. Acad. Sci. Ukraine, N5, 40 (1994).

[22] R.Z.Zhdanov, J. Phys. A: Math. Gen., 28, 3841 (1995).

[23] A.S. Fokas and Q.M. Liu, Phys. Rev. Lett., 72, 3293 (1994).

[24] W.I. Fushchych and A.G. Nikitin, Symmetries of Equations of Quantum Mechanics, (Allerton Press, New York, 1994).

[25] A.G.Nikitin, J. Non. Math. Phys., 2, 405 (1995).

[26] O.V. Kaptsov, Non. Anal., Theory, Meth. Appl., 19, 753 (1992).

[27] A. Gonsalez-Lopez, N. Kamran and P. Olver, Comm. Math. Phys., 153, 117 (1993).

[28] W.I. Fushchych and R.Z. Zhdanov, Sov. J. of Part. and Nuclei, 19, 1154 (1988).

[29] R.Z. Zhdanov, Preprint ASI-TPA/23/95, Technical University of Clausthal (1995).

[30] E. Kamke, Differentialgleichungen Lösungmethoden und Lösungen (Akademische Verlagsgesellschaft, Leipzig, 1976).

[31] A.G. Nikitin, S.P. Onufriychuk and W.I. Fushchych, Theor. Math. Phys., 91, 268 (1992). 
[32] N.Kh. Ibragimov, Transformation Groups Applied to Mathematical Physics (Reidel, Dordrecht, 1985).

[33] A.K. Grant and J.L. Rosner, J. Math. Phys., 35, 2142 (1994).

[34] W. Miller, Symmetry and Separation of Variables (Addison-Wesley, Massachusetts, 1977).

[35] R.Z. Zhdanov, I.V. Revenko and W.I. Fushchych, J. Math. Phys., 36, 5505 (1995).

[36] H.-D. Doebner and G.A. Goldin, J. Phys. A: Math. Gen., 27, 1771 (1994).

[37] P. Nattermann, Rep. Math. Phys., 36, 387 (1995). 